\title{
Quasi-Digital Biosensor-Interface for a Portable Pen to Monitor Anaesthetics Delivery
}

\author{
Simone Aiassa*†, Francesca Stradolini ${ }^{\dagger}$, Abuduwaili Tuoheti* ${ }^{* \dagger}$, Sandro Carrara ${ }^{\dagger \ddagger}$, and Danilo Demarchi* ${ }^{* \ddagger}$ \\ *Department of Electronics and Telecommunications (DET), Politecnico di Torino, Turin, Italy \\ ${ }^{\dagger}$ Integrated Systems Laboratory (LSI), École Polytechnique Fédérale de Lausanne (EPFL), Lausanne, Switzerland \\ ${ }_{\ddagger}^{\ddagger}$ Integrated Circuits Laboratory (ICLAB), École Polytechnique Fédérale de Lausanne (EPFL), Neuchâtel, Switzerland \\ Email: simone.aiassa@polito.it
}

\begin{abstract}
Monitoring of patient response to the anaesthetic drugs is an attractive improvement for achieving a correct balance of sedation level, increasing the chance of success in the right procedure of anaesthesia. Nowadays, there are no commercial tools able to offer real-time monitoring of anaesthetics, indeed, there is still a lack in sensing technologies able to maintain high performances in long term monitoring within a portable miniaturised hardware system. To overcome these limitations, we are here presenting the innovative concept of a portable pen-device able to sense anaesthetic compounds over time. This study is based on an electrochemical sensor to be fully integrated into a complete pen-shaped point-of-care for the monitoring of anaesthesia delivery. The design of the system is based on a bio-inspired event-based approach that is guaranteeing low complexity, low power consumption and is therefore suitable to be scaled to fit the barrel of a pen. An exhaustive comparison between the proposed system and a lab instrument proves that the presented approach obtains comparable performances in terms of sensitivity and resolution with the ones obtained by expensive commercial instrumentation, meanwhile, the results show a $95 \%$ power consumption reduction and a $92 \%$ area decrease w.r.t. previously presented implementation.
\end{abstract}

Index Terms-Anaesthesia, bio-inspired, biosensor, portable, quasi digital, therapeutic drug monitoring.

\section{INTRODUCTION}

The anaesthesia is a critical surgery medical procedure that requires the administration of several drugs: typically, hypnotic, analgesic, and muscle relaxant. To obtain appropriate sedation and avoid intoxication, the delivery of these compounds must be balanced and accurate. The Depth Of Anaesthesia (DOA) is the metric to express the effect of anaesthesia on the patient and it is nowadays evaluated by the estimation of the Bispectral Index (BIS), which is a weighted sum of several ElectroEncephaloGraphic (EEG) parameters [1]. However, this approach presents some limitations and can lead to wrong dose estimation due to the high inter-patient variability [2]. To improve the traditional approach of anaesthesia delivery, this work aims to present a Therapeutic Drug Monitoring (TDM) system able to provide on-line monitoring of the actual drug concentration infused in the patient's veins. This will allow a dynamic and personalised adjustment of the drug delivery [3].

Electrochemical investigation can be adopted to measure the therapeutic compounds, in particular, Cyclic Voltammetry

This work is supported by Politecnico di Torino and Compagnia di San Paolo under the initiative "Joint research projects with top universities".
(CV) and Differential Pulse Voltammetry (DPV) [4] are highly attractive method due to fast measurements, multi-sensing, and high sensitivity [5]. In voltammetry, a voltage sweep is applied to an electrochemical cell across its Working Electrode (WE) and its Reference Electrode (RE). The applied potential makes a Redox reaction to happen on the electrochemical sensor and the current is then measured from the Counter Electrode (CE). The resulting waveform (current upon applied voltage) shows peaks, which give a reliable measure of the actual drug concentration, and this can be done by systems called potentiostats. Architectures able to monitor the anaesthetic delivery have been recently presented, however, they are still not easily integrable into portable or wearable devices [6], [7].

The focus of this paper is the design, development, and validation of a Commercial-Off-the-Shelf (COTS) componentbased potentiostat, whose size is comparable with a pen. The major design novelty of the proposed architecture is the amperometric sensing through bio-inspired event-based techniques, which is innovative with respect to the standard approach involving power-hungry DAC and ADC in the architecture [8]. Indeed, the full proposed potentiostat is a quasi-digital electronic interface. In the quasi-digital domain, the information is coded in timing in a series of pulses, called events, containing both the properties of analog and digital signals. This event-based design approach presents indeed great advantages considering the processing in the time domain [9], the noise-

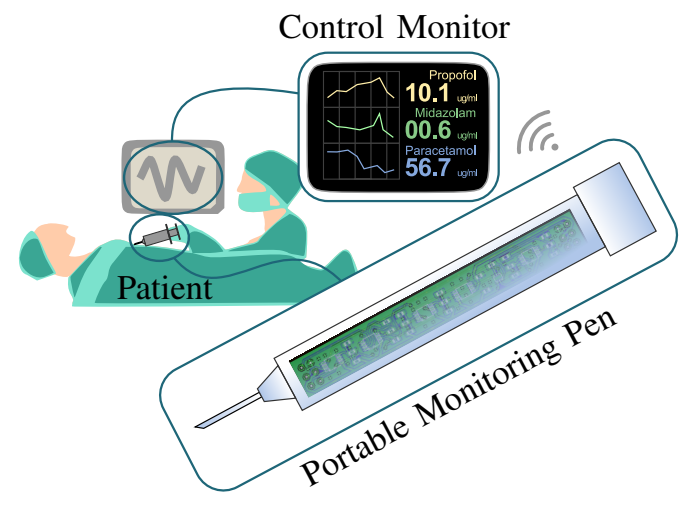

Fig. 1: Concept of the proposed system, the portable monitoring pen will provide in real-time the concentration of the drugs infused in the patient under anaesthesia. 
reduction, and the accuracy increase [10]. The compact readout circuit is reducing both size and power consumption, the information translation is continuous, without informationloss, with a lower transmission energy cost [11]. The literature presents numerous examples of applications based on bioinspired and quasi-digital approaches [12]-[14], although, to the best of our knowledge, they have never been integrated for the scope of providing on-line monitoring of anaesthesia.

The proposed architecture has been validated by comparing the measurement results with the ones achieved by a commercial laboratory potentiostat. The acetaminophen (APAP) is the benchmark drug since it is usually administered in anaesthesiology [15].

\section{THE CONCEPT}

We propose here a small and portable object able to monitor the anaesthetic within a pen-shape as shown in Fig. 1. The object shall be considered as an additional instrument to give the anaesthesiologist the ability to continuously monitor the drug absorption in the patient. The system must contain an electronic potentiostat (composed by a voltage driver and a current read-out) and an electrochemical sensor for anaesthetic sensing. Fig. 2 presents the developed prototype of the portable pen. In $18 \mathrm{~mm}$ diameter plastic tube a custom Printed Circuit Board (PCB) implements the proposed architecture, while a commercial Screen-Printed Electrode (SPE) acts as an electrochemical sensor for the APAP.

\section{DESIGN AND IMPLEMENTATION}

The presented bio-sensor electronic-interface relies on quasi-digital to analog conversion, and vice-versa. Fig. 3 shows the proposed design which contains a voltage driver $(P W M t o V)$ and a current read-out (ItoQDE). The voltage follower (VF) avoids current flow on the RE. The grounded WE is the selected topology for the potentiostat architecture since it minimises the number of components required [4]. The WE is connected to half-supply $\left(1 / 2 \mathrm{~V}_{\mathrm{DD}}\right)$ to achieve both dual-voltage driving and dual-current sensing (i.e. driving and measuring both positive and negative voltages/currents) without the introduction in the system of a dual-voltage supply.

To drive the electrochemical cell a Pulse Width Modulated (PWM) signal (which is indeed a type of q.d. signal) is converted to a stable voltage by the PWMtoV. This block is a second-order active low-pass filter in the Sallen-Key configuration. The driving potential applied between the $\mathrm{CE}$ and the

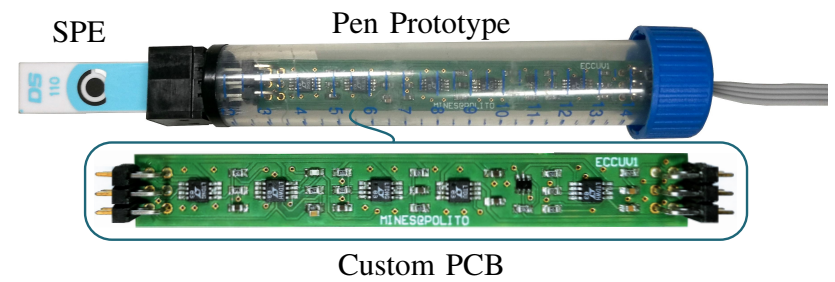

Fig. 2: Portable pen, the custom PCB implements the proposed potentiostat and the SPE acts as electrochemical sensor.

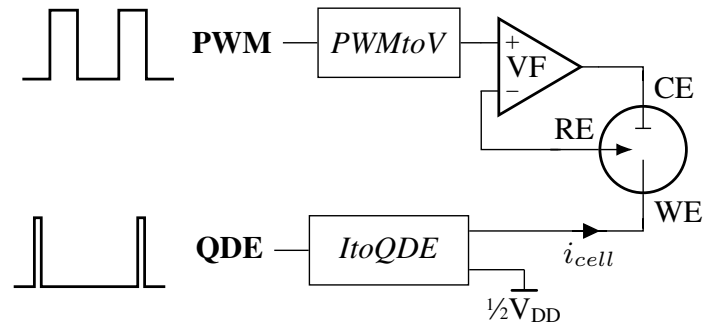

Fig. 3: Proposed potentiostat, the voltage driver $(P W M t o V)$ and the current read-out (ItoQDE) are both event-based modules.

WE is so directly proportional to the Duty Cycle (DC) of the PWM input signal. At convenience, it is possible to furnish to the electrochemical cell an increasing or decreasing voltage ramp, just modulating the $\mathrm{DC}$ of the input. The oxidation or reduction current $\left(i_{\text {cell }}\right)$ is read converting it to a Quasi-Digital stream of Event (QDE) by the ItoQDE block. Differently from what the literature proposed in [12], [16], [17], we show here a low-complexity one-input one-output read-out circuit able to convert the dual-range current in an event stream, with a single-voltage supply. The Fig. 4 shows the schematic of the Ito $Q D E$ module which contains three amplifier blocks: a transimpedance $\left(A_{1}\right.$ and $\left.R_{1}\right)$, an integrator $\left(A_{2}, R_{2}\right.$, and $\left.C_{1}\right)$ and a comparator $\left(A_{3}, R_{4}\right.$, and $\left.R_{5}\right)$. Fig. 5 presents the timing diagram of the current to frequency conversion. The capacitor $\mathrm{C}_{1}$ is discharged according to the input current (adapted to the dynamic by the trans-impedance amplifier), the comparator notifies when $\mathrm{V}_{\mathrm{C} 1}$ overcomes the lower threshold, this sets QDE. After that, a reference current rapidly charges again the capacitor through the feedback MOSFET $\left(\mathrm{M}_{1}\right.$ and $\left.\mathrm{R}_{3}\right)$, the signal QDE is reset, and the conversion can start again. The sampled current $\left(i_{\text {cell }}\right)$ is proportional to the timing between two consecutive edges according to (1), where $i_{0}$ is the offset

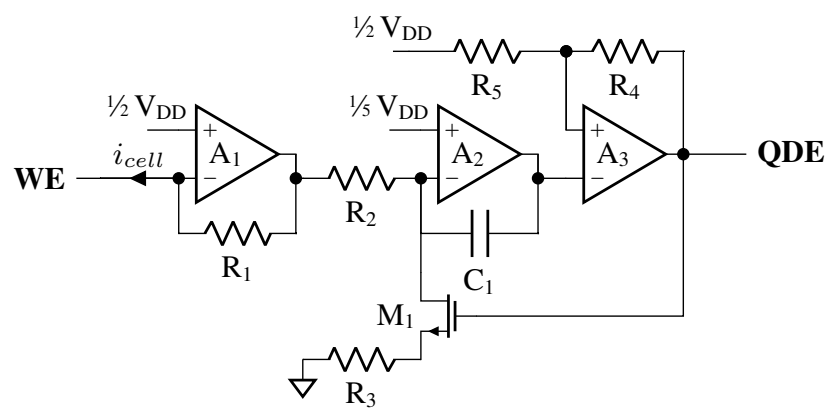

Fig. 4: Scheme of the ItoQDE converter. Current from the WE (right) is converted to QDE train of pulses (left).

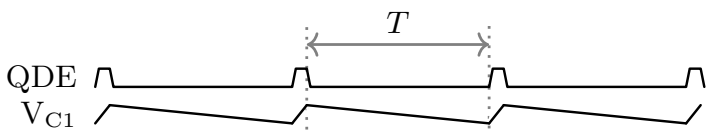

Fig. 5: Ito $Q D$ converter timing diagram. The timing $(T)$ of QDE signal is proportional to the monitored current. 
current and $\mathrm{V}_{\text {th }}$ is the comparator threshold.

$$
i_{\text {cell }}=-\frac{\mathrm{R}_{2} \cdot \mathrm{C}_{1} \cdot \mathrm{V}_{\mathrm{th}}}{\mathrm{R}_{1} \cdot T}+i_{0}
$$

In the implemented potentiostat the PCB size is $78 \times 10 \mathrm{~mm}$. Tuning the component according to Eq. 1, the driving voltage range is set to $[-1.2: 1.2] \mathrm{V}$, while the input current range is $[-60: 60] \mu \mathrm{A}$. The system is designed to fit an input PWM signal at the frequency of $20 \mathrm{kHz}$. In the current range, the event rate (i.e. frequency of the events) of the output QDE signal spans across $[0.38: 34] \mathrm{kevent} / \mathrm{s}$, with a resting event rate of $17 \mathrm{kevent} / \mathrm{s}$. A closed feedback loop experiment is performed to verify the system, inserting a resistor between shortened CE-RE and WE nodes. Our experiments highlighted that both PWM to voltage and current to QDE conversions are linear with a maximum linearity error of $0.14 \%$. In order to quantify the noise level, the WE input is left unconnected and the current is sampled at the operating frequency of the $\mathrm{CV}$, namely $60 \mathrm{~Hz}$. Considering the standard deviation of the samples, the level of noise is $39 \mathrm{nA}$. In the full scale $( \pm 60 \mu \mathrm{A})$, the equivalent number of bits is $11.6 \mathrm{bit}$, closer to the resolution of DAC usually implemented in a custom-built potentiostat for bio-sensing application (12 bit [5]). The mean total power consumption of the board measured during lab testing is $19.5 \mathrm{~mW}$.

\section{SySTEM VALIDATION}

As visible in Fig. 2, the potentiostat described in Section III has been interfaced to the electrochemical sensor for the APAP. This sensor has been obtained by carbon Screen-Printed Electrode (SPE), namely DropSens DRP-110, with a central carbon WE (4 mm diameter), carbon CE, and silver RE. To perform the tests, APAP powder from Sigma Aldrich ${ }^{\circledR}$ is dissolved in Phosphate Buffer Saline (PBS, pH 7.4, $10 \mathrm{mM}$ ). The PBS is also used as background electrolyte. The measure is performed on subsequent increasing concentration of APAP in the therapeutic range $[50: 300] \mu \mathrm{M}$ with an increase step of $50 \mu \mathrm{M}$. The measure is repeated three times for each condition with a new electrode to consider the inter-electrode variability. Two different electrochemical sensing methods are taken into account: CV and DPV. The CV is performed with a Scan Rate $(\mathrm{SR})$ of $0.2 \mathrm{~V} / \mathrm{s}$, in the range $[-0.1: 1.1] \mathrm{V}$, with a voltage step of $6 \mathrm{mV}$, and a time step of $30 \mathrm{~ms}$. The DPV is performed at $0.1 \mathrm{~V} / \mathrm{s}$, in the range $[0: 1] \mathrm{V}$, with a voltage step of $6 \mathrm{mV}$, a time step of $60 \mathrm{~ms}$, the modulation amplitude is set to $60 \mathrm{mV}$, and the modulation time to $30 \mathrm{~ms}$. The difference in SR between the two method is introduced in order to compensate the higher sensitivity of DPV method [4]. An FPGA board Xilin ${ }^{\circledR}$ Artix $-7^{\mathrm{TM}}$ controls the realised system. A custom-built digital interface generates the PWM signal to drive the cell with the waveform required by the voltammetry technique. Measuring the timing of the event received on the QDE line with a simple free-running counter, the current is constantly sampled every voltage step. The data collected are sent out through a UART-USB port and stored.
To validate our system, the sensing of APAP has been also repeated by using a lab instrument, i.e. the Metrohm Autolab PGSTAT 302N driven by the software Nova 1.11. The data received both from the Autolab and from the developed system are processed in Matlab $^{\circledR}$ (v. R2017b), without any further post-processing or filter. The peaks are detected with the findpeaks built-in function, after a blank-baseline rigid translation. The function returns prominence of the current peak, this value is considered for the final estimation of the drug concentration.

\section{Results}

Fig.6 displays a comparison in the output acquired by the two devices (our system and Autolab) considering CV (6a, 6b) and DPV $(6 \mathrm{c}, 6 \mathrm{~d})$. The proposed solution introduces a certain level of white noise $(6 \mathrm{~b}, 6 \mathrm{~d})$, despite this, in both methods the results are comparable. Fig. 7 reports the calibration curves obtained by the analysis of the data. The implemented system does not introduce a visible reduction in sensitivity, the two fitting curves almost overlap with both methods. The evaluated regression coefficient $\left(\mathrm{r}^{2}\right.$, higher than 0.99) does not change, hence, the system does not introduce non-linearities. Table I presents a comparison between the two architectures. The sensitivity is similar ( $-12 \%$ w.r.t. Autolab in CV), with an uncertain $20 \%$ lower in the presented solution (w.r.t. Autolab in DPV). Even if the Limit Of Detection (LOD, the minimum detectable quantity of analyte, computed as three times the standard deviation of the blank around the peak, divided by

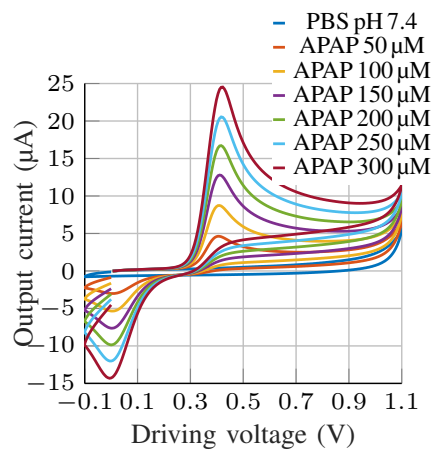

(a) Autolab CV

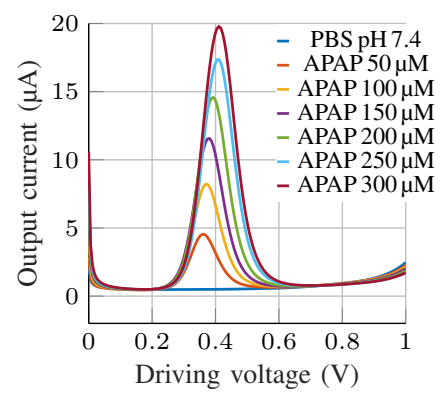

(c) Autolab DPV

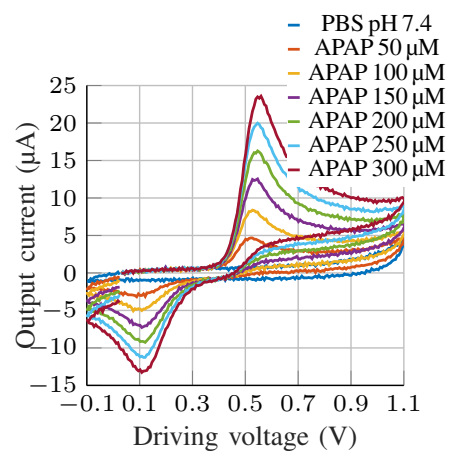

(b) Our system CV

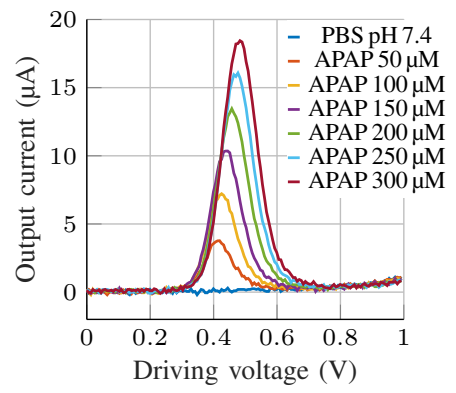

(d) Our system DPV
Fig. 6: Output voltammograms obtained by Autolab (left) and by our system (right), considering CV (top) and DPV (bottom). 


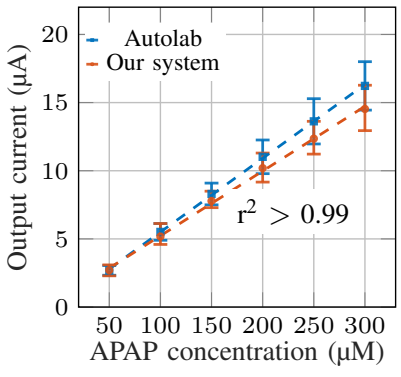

(a) $\mathrm{CV}$

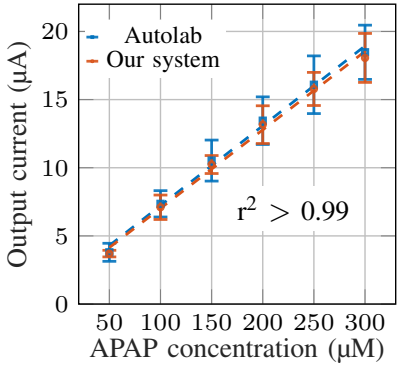

(b) DPV
Fig. 7: Calibration curves comparison between Autolab and our system, for CV (a) and DPV (b).

TABLE I: Results comparison between Autolab and our system, considering CV and DPV.

\begin{tabular}{lcccc}
\hline & \multicolumn{2}{c}{ CV } & \multicolumn{2}{c}{ DPV } \\
& Autolab & Our system & Autolab & Our system \\
\hline $\begin{array}{l}\text { Sensitivity } \\
(\mathrm{nA} / \mu \mathrm{M})\end{array}$ & $54.1 \pm 7.1$ & $47.5 \pm 5.0$ & $58.6 \pm 5.9$ & $57.6 \pm 5.6$ \\
LOD $(\mu \mathrm{M})$ & $1.43 \pm 0.18$ & $5.43 \pm 0.57$ & $0.57 \pm 0.06$ & $5.08 \pm 0.49$ \\
\hline
\end{tabular}

the sensitivity) has a higher value in our system, due to lower noise rejection, the value remains one order of magnitude lower than the minimum therapeutic concentration of the APAP, hence, confirming its excellent performance. These findings are highly promising, considering the reduction of three orders of magnitude of hardware cost, the minimal size, and the portability between our system under test and the commercial instrumentation. The results prove that the presented approach does not require the introduction of postprocessing, analog/digital filter, and differential measurement in our system, methods all adopted by the Autolab, to get comparable performance.

Table II shows a comparison between our solution and other anaesthetic sensing circuits presented in literature [5], [7]. Our system is suitable for the target application (as demonstrated by the comparison w.r.t Autolab) with the advantage of removing ADC and DAC, featuring $5 \%$ of the power consumed by [7] in $8 \%$ of the size of [5].

\section{CONCLUSION}

We presented a novel concept and newly-designed architecture for drug monitoring targeted to be inserted in a portable pen for improved anaesthesiology practice. The results prove that our approach provides a portable, small, and low power system (respectively $92 \%$ and $95 \%$ reduction w.r.t. previous

TABLE II: Comparison with anaesthetic sensing systems.

\begin{tabular}{lccc}
\hline & {$[5]$} & {$[7]$} & Our system \\
\hline Conversion type & ItoV & ItoV & ItoF \\
No of ADCs/DACs & 2 & 2 & 0 \\
Board size $\left(\mathrm{mm}^{2}\right)$ & 9600 & n.a. & 780 \\
Power Consumption $(\mathrm{mW})$ & n.a. & 390 & 19.5 \\
\hline
\end{tabular}

implementations), always maintaining comparable sensitivity with respect to reference commercial lab instrumentations, and LOD one order of magnitude below the requested minimum therapeutic concentration. Other anaesthetics, such as midazolam and propofol are potentially detectable by this method, therefore future works will test and validate performance in monitoring these anaesthetics Moreover, the future steps are the design of a full custom digital interface (optimised for the bio-inspired event-based processing), and the development of a needle-shaped bio-sensor.

\section{REFERENCES}

[1] A. Arslan, B. Şen, F. V. Çelebi, M. Peker, and A. But, "A comparison of different classification algorithms for determining the depth of anesthesia level on a new set of attributes," in 2015 Int. Symp. on Innovations Intelligent Systems Appl. (INISTA), pp. 1-7, Sept 2015.

[2] Z. Hajat, N. Ahmad, and J. Andrzejowski, "The role and limitations of EEG-based depth of anaesthesia monitoring in theatres and intensive care," Anaesthesia, vol. 72, no. S1, pp. 38-47.

[3] A. Simalatsar, M. Guidi, P. Roduit, and T. Buclin, "Robustness analysis of personalised delivery rate computation for IV administered anesthetic," Smart Health, 2018.

[4] S. Carrara, Bio/CMOS Interfaces and Co-Design. New York: SpringerVerlag, 2013

[5] F. Stradolini, T. Elboshra, A. Biscontini, G. De Micheli, and S. Carrara, "Simultaneous monitoring of anesthetics and therapeutic compounds with a portable multichannel potentiostat," in 2016 IEEE Int. Symp. on Circuits Syst. (ISCAS), pp. 834-837, May 2016.

[6] F. Stradolini, A. Tuoheti, P. Motto Ros, D. Demarchi, and S. Carrara, "Raspberry Pi based system for portable and simultaneous monitoring of anesthetics and therapeutic compounds," in 2017 New Generation CAS (NGCAS), pp. 101-104, Sept 2017.

[7] F. Stradolini, A. Tuoheti, T. Kilic, S. L. Ntella, N. Tamburrano, Z. Huang, G. De Micheli, D. Demarchi, and S. Carrara, "An IoT solution for online monitoring of anesthetics in human serum based on an integrated fluidic bioelectronic system," IEEE Trans. on Biomed. Circuits Syst., pp. 01-09. 2018.

[8] P. Motto Ros, M. Crepaldi, A. Damilano, and D. Demarchi, "Integrated bio-inspired systems: An event-driven design framework," in 2014 10th Int. Conf. on Innovations in Inf. Technol. (IIT), pp. 48-53, IEEE, Nov 2014.

[9] N. V. Kirianaki, S. Y. Yurish, N. O. Shpak, and V. P. Deynega, Signal Processing in Quasi-Digital Smart Sensors, ch. 6, pp. 129-142. WileyBlackwell, 2002.

[10] O. L. Nuzumlali, "Detailed noise analysis of current-to-frequency converters for precision analog accelerometers," in 2016 IEEE/ION Position, Location and Navigation Symp. (PLANS), pp. 898-904, Apr 2016.

[11] P. Motto Ros, B. Miccoli, A. Sanginario, and D. Demarchi, "Low-power architecture for integrated CMOS bio-sensing," in 2017 IEEE Biomed. Circuits Syst. Conf. (BioCAS), pp. 1-4, Oct 2017.

[12] H. S. Narula and J. G. Harris, "A time-based VLSI potentiostat for ion current measurements," IEEE Sensors J., vol. 6, pp. 239-247, Apr 2006.

[13] S. Sapienza, M. Crepaldi, P. Motto Ros, A. Bonanno, and D. Demarchi, "On integration and validation of a very low complexity ATC UWB system for muscle force transmission," IEEE Trans. on Biomed. Circuits Syst., vol. 10, pp. 497-506, Apr 2016.

[14] S. Aiassa, P. Motto Ros, G. Masera, and M. Martina, "A low power architecture for AER event-processing microcontroller," in 2017 IEEE Biomed. Circuits Syst. Conf. (BioCAS), pp. 1-4, Oct 2017.

[15] H. Khouadja, H. Arnous, K. Tarmiz, D. Beletaifa, A. Brahim, W. Brahem, J. Sakhri, and K. Ben Jazia, "Pain on injection of propofol: Efficacy of paracetamol and lidocaine," Open J. of Anesthesiology, vol. 4, no. 4, pp. 81-87, 2014.

[16] M. M. Ahmadi and G. A. Jullien, "Current-mirror-based potentiostats for three-electrode amperometric electrochemical sensors," IEEE Trans. Circuits Syst. I, Reg. Papers, vol. 56, pp. 1339-1348, July 2009.

[17] N. Qiao and G. Indiveri, "An auto-scaling wide dynamic range current to frequency converter for real-time monitoring of signals in neuromorphic systems," in 2016 IEEE Biomed. Circuits Syst. Conf. (BioCAS), pp. 160163, Oct 2016. 